\title{
Efectos de las causas de mortalidad adulta en la esperanza de vida, entre 1985 y 1999, según regiones colombianas
}

\author{
Elizabeth López ${ }^{1}$, Patricia Arce² \\ ${ }^{1}$ Grupo Sociedad y Salud, Subdirección de Investigación, Instituto Nacional de Salud, Bogotá, D.C., Colombia \\ ${ }^{2}$ Área de Vigilancia en Salud Pública, Secretaría de Salud de Bogotá, Bogotá, D.C., Colombia \\ Introducción. El estudio de la mortalidad por causas es fundamental para conocer el estado \\ de salud de una población. \\ Objetivos. Establecer los cambios de la mortalidad por causas y su contribución a la esperanza \\ de vida de la población de 15 a 74 años entre 1985 y 1999, según sexo y región. \\ Materiales y métodos. Estudio descriptivo retrospectivo; las fuentes de información fueron las \\ defunciones registradas de 1983 a 2001 y las proyecciones de población del Departamento \\ Administrativo Nacional de Estadística. Los cambios en la mortalidad se midieron utilizando la \\ metodología de Eduardo E. Arriaga, que se basa en el cálculo de la esperanza de vida temporal, \\ los índices de cambio absoluto y relativo, y la contribución del cambio de la mortalidad por \\ causa de muerte a la esperanza de vida. \\ Resultados. La principal causa de la pérdida en la esperanza de vida temporal en ambos \\ sexos fue el incremento de las defunciones por suicidios, homicidios y otras causas violentas, \\ aunque la pérdida fue mayor para los hombres que para las mujeres en todas las regiones \\ estudiadas. El mayor aporte positivo se logró por la disminución de las enfermedades del \\ sistema circulatorio y los accidentes. \\ Conclusión. La ganancia en la esperanza de vida temporal fue mínima puesto que los aportes \\ positivos producidos por la disminución de la mortalidad por causas naturales se anulan con \\ los aportes negativos producidos por el incremento de la mortalidad por suicidios, homicidios \\ y otras violencias que son evitables.
}

Palabras clave: tasa de mortalidad, causa de muerte, causas externas, esperanza de vida, tablas de vida, Colombia

A comparison of the causes of adult mortality and its effects on life-expectancy across the regions of Colombia

Introduction. When determining some population's state of health, an understanding of the causes of mortality is essential.

Objectives. Changes in mortality due to causes was established to determine their contribution to the life-expectancy by gender and region of the Colombian population aged 15 to 74, between 1985 and 1999, by gender and region.

Materials and methods. This was a descriptive, retrospective study; the sources of information were records of deaths from 1983 to 2001 and population projections according to Departamento Administrativo Nacional de Estadística. The age selected as a sample population was 15 to 74 . Changes in mortality were measured by using Eduardo Arriaga's methodology, which is based on calculating temporary life-expectancy, absolute and relative change indices, and how changes in mortality due to cause of death contribute to life-expectancy.

Results. The main cause of reduced temporary life-expectancy in both genders was the increase in deaths by suicide, homicide and other violent causes (the reduction was greater for men than women in all regions studied). The greatest positive contribution to longevity was by the reduction in circulatory system diseases and accidents.

Conclusions. A minimal gain in temporary life-expectancy was achieved as the positive affect of reduced mortality due to natural causes. This gain was annulled by the negative contributions of increased mortality due to suicide, homicide and other violent avoidable acts.

Key words: mortality rate, cause of death, external causes, life expectancy, life tables, Colombia 
El estudio de la mortalidad por causas es fundamental para conocer el estado de salud de una población, razón por la cual es necesario identificar las principales causas de muerte que están afectando a la población, para que se definan programas de promoción de la salud y prevención de la mortalidad acordes con las necesidades de cada región.

La esperanza de vida al nacer se utiliza a menudo como una medida resumen de la mortalidad de una población y los cambios en la esperanza de vida se usan también para resumir los cambios en la mortalidad de una población (1).

La esperanza de vida al nacer se ha incrementado en la mayoría de los países de América, debido principalmente a la reducción de la mortalidad en menores de 15 años, y en la población adulta se ha reducido, pero no como se esperaría, particularmente para los hombres, pero en algunos países, como Barbados, Colombia, Costa Rica, Cuba, Ecuador y Puerto Rico, se incrementó en algunos grupos de edad entre los comienzos de los 80 y mediados de los 90 (2).

La muerte prematura tiene un alto costo social y, por lo tanto, su análisis es de la mayor relevancia para evaluar la situación de salud de las poblaciones. Las tasas de mortalidad permiten conocer la probabilidad de morir y, por ello, constituyen un insumo básico para el análisis epidemiológico. En las dos últimas décadas del siglo XX, en la región de las Américas se ha observado una reducción de la probabilidad de morir cercana al $25 \%$ y, como consecuencia, un aumento promedio en la esperanza de vida al nacer de casi seis años.

En Colombia, la esperanza de vida al nacer para los hombres se incrementó en 2,81 años al pasar de 64,3 a 67,1 , mientras que para las mujeres

\footnotetext{
Correspondencia:

Elizabeth López, Grupo Sociedad y Salud, Subdirección de Investigación, Instituto Nacional de Salud, Avenida Calle 26 № 51-20, zona 6, CAN, Bogotá, D.C., Colombia.

Teléfono: 2207700 , extensión 321; fax: 2207700 , extensiones 255 y 310

elopez@ins.gov.co

Recibido: 07/12/07; aceptado:23/07/08
}

pasó de 70,1 a 74,2 incrementándose en 4,14 años, entre comienzos de 1980 y finales de 1990; este incremento se debe, en parte, a la reducción de la mortalidad infantil; sin embargo, el gran incremento de la mortalidad adulta por causas violentas ha producido un efecto negativo mayor en la esperanza de vida de los hombres que en la de las mujeres (3).

En casi todos los países de América Latina y el Caribe la mortalidad por causas externas, las enfermedades del sistema circulatorio y los tumores son las principales causas de muerte en los hombres adultos, mientras que en las mujeres son las enfermedades del sistema circulatorio, los tumores y las causas externas; sin embargo, las diferencias entre hombres y mujeres son mucho mayores en Colombia que en otros países (2).

Se realizó este estudio con el objetivo de establecer los cambios presentados en la mortalidad por causas entre 1985 y 1999 y su contribución a la esperanza de vida en la población de 15 a 74 años según sexo y región, teniendo en cuenta que la situación de la mortalidad no es la misma en todo el país puesto que las condiciones económicas, sociales, culturales y ambientales no son las mismas en todas las regiones por las diferencias y brechas entre poblaciones en el acceso a los servicios y a la salud, según área (rural-urbana), sexo, edad, grupos étnicos, migrantes, etc. (4).

\section{Materiales y métodos}

Es un estudio descriptivo retrospectivo; las fuentes de información fueron los registros de defunción de los años 1983 a 2001 y las proyecciones de población del Departamento Administrativo Nacional de Estadística (DANE). Con el fin de evitar las variaciones anuales, las defunciones para 1985 y 1999 se obtuvieron promediando las defunciones de los quinquenios 1983-1987 y 1997-2001, respectivamente. Teniendo en cuenta la baja cobertura de las estadísticas vitales en algunos departamentos, fue necesario el ajuste de las defunciones con base en las defunciones estimadas (5), conservando la estructura por edad y causa y el índice de masculinidad de las defunciones registradas, para lo cual se calculó el promedio 
de defunciones por grupos de edad, causa y sexo para cada uno de los quinquenios mencionados.

Las proyecciones de población se utilizaron para construir las tablas de mortalidad y como denominadores para el cálculo de las tasas de mortalidad.

Las causas de muerte se agruparon de acuerdo a la metodología propuesta por Eduardo Arriaga (6), que establece los siguientes grupos: enfermedades infecciosas y parasitarias, tumores (malignos y benignos), enfermedades del sistema circulatorio, neumonía y bronquitis, enfermedades del sistema digestivo, accidentes, suicidios, homicidios y otras causas violentas excluyendo accidentes, la mortalidad materna, las mal definidas y causas desconocidas y el resto. Para realizar esta agrupación se utilizó la equivalencia de causas de defunción de la Clasificación Estadística Internacional de Enfermedades y Problemas Relacionados con la Salud, novena revisión (CIE9) a la Clasificación Estadística Internacional de Enfermedades y Problemas Relacionados con la Salud, décima revisión (CIE-10), elaborada por el DANE, teniendo en cuenta que en los años 1985 a 1996 la causa básica estaba codificada con la CIE-9, y los años 1997 a 2001 con la CIE-10 $(7,8)$.

Para el estudio se incluyeron las defunciones de 15 a 74 años y las variables analizadas fueron sexo y región. Cada región está conformada por los siguientes departamentos:

Atlántica: Atlántico, Bolívar, Cesar, Córdoba, La Guajira, Magdalena y Sucre.

Oriental: Boyacá, Cundinamarca, Meta, Norte de Santander y Santander.

Central: Antioquia, Caldas, Caquetá, Huila, Quindío, Risaralda y Tolima.

Pacífica: Cauca, Chocó, Nariño y Valle.

Nuevos departamentos: Arauca, Casanare, Putumayo, San Andrés, Amazonas, Guainía, Guaviare, Vaupés y Vichada.

Para medir los cambios en la mortalidad por sexo y grupo de causas se utilizó la metodología desarrollada por Eduardo E. Arriaga (6), que está basada en el cálculo de los siguientes indicadores, a partir de la construcción de las tablas de vida para dos momentos (1985 y 1999):

- Esperanza de vida temporal: mide el número promedio de años que se vive, entre dos edades exactas, bajo un nivel determinado de mortalidad.

El número de años vividos se mide por la función $L_{x}$ de la tabla de mortalidad. Esta función se puede interpretar como el número de años vividos entre la edad $x$ y $(x+1)$ por aquellos nacidos en el mismo instante (esto es, la raíz de la tabla, o sea, $I_{0}$ ) bajo condiciones de mortalidad constantes. Usando el número acumulado de años que se van a vivir desde la edad $x$ hasta el final de la vida (edad w), esto es,

$$
T_{x}=\sum_{i=0}^{w} L_{x+i}
$$

y el número de personas vivas a una edad exacta $x\left(I_{x}\right)$; es posible definir la esperanza de vida temporal a la edad $x$ como:

$$
e_{x}=\frac{T_{x}-T_{x+n}}{I_{x}}
$$

- Índice de cambio absoluto $\left({ }_{n} A C_{x}\right)$ : es simplemente la diferencia entre dos esperanzas de vida temporales para los mismos grupos de edad, pero bajo condiciones distintas de mortalidad. Si las tablas de mortalidad, en las que se reflejan las condiciones de mortalidad de la población, fueron construidas en los años t y $(t+i)$, el índice de cambio absoluto sería:

$$
{ }_{n} A C_{x}^{t, t+i}=e_{x}^{t+i}-e_{x}^{t}
$$

donde el sobreíndice indica el año. El índice ${ }_{n} A C_{x}, t+i$ mide el cambio en el número promedio de años a ser vividos entre las edades $x$ y $(x+n)$ para aquellas personas vivas a la edad exacta $x$, debido al cambio de la mortalidad entre los años $t$ y $(t+i)$.

- Índice de cambio relativo $\left({ }_{n} R C_{X}{ }^{t, t+1}\right)$ : el índice de cambio relativo muestra el aumento en el número promedio de años a ser vividos entre la edad $x$ y $(x+n)$ para aquellas personas vivas a la edad exacta $x$, con relación al aumento "posible" de la esperanza de vida temporal en el año t. En símbolos sería: 


$$
{ }_{n} R C_{x}^{t, t+i}=\frac{{ }_{n} A C_{X}^{T, t+i}}{n-e_{X}^{t}}
$$

donde $n$ es el número de años por ser vividos entre la edad $x$ y $(x+n)$ si nadie muriera.

- La contribución del cambio de la mortalidad por causa de muerte a la esperanza de vida en cada grupo de edad, se estima bajo el supuesto de que es proporcional a la contribución del cambio de la tasa central de mortalidad. Para medir la contribución en cada grupo de edad, se estima el número de años adicionados o perdidos en la esperanza de vida, a causa de la disminución o aumento, respectivamente, de la tasa central de mortalidad de la tabla de vida (índice de cambio absoluto); la contribución en la esperanza de vida debida al cambio en la mortalidad de un grupo de edad específico por la causa de muerte $z$ será $(8)$ :

donde:

$$
{ }_{n} A C_{x}(z)={ }_{n} A C_{x} x \frac{{ }_{n} C M_{x}(z)}{{ }_{n} C M_{x}}
$$

${ }_{n} C M_{x}={ }_{n} m_{x}^{2}{ }_{x n} m_{x}^{1}$ es la diferencia entre las tasas específicas de mortalidad de los dos períodos en el grupo $(x, x+n)$, y mide el cambio total experimentado por la mortalidad de ese grupo entre dos períodos.

${ }_{n} C M_{x}(z)={ }_{n} m_{x}{ }_{x}(z)-{ }_{n} m_{x}^{1}(z)$ es el cambio en la mortalidad por la causa de muerte $z$.

Este método permite medir la contribución de cada causa de muerte o grupo de edad al cambio observado en la esperanza de vida; el cambio se expresa en años de esperanza de vida que son positivos con la reducción de la mortalidad y negativos con el incremento de la mortalidad.

Este método tiene tres ventajas: 1) considera el hecho de que los cambios similares en las tasas de mortalidad a edades diferentes influencia de manera distinta la esperanza de vida; 2 ) da cuenta de la sustitución entre causas de muerte comparativas, y 3) proporciona una medida cuantitativa de fácil interpretación. Tiene la desventaja relativa de ser sensible a los valores extremos del nivel basal de mortalidad por dicha causa, lo que significa que, si la mortalidad por una causa ya hubiera alcanzado un nivel muy bajo, su posterior reducción representa una contribución pequeña en años de esperanza de vida ganados; mientras que si su nivel basal fuera muy alto, cualquier reducción produce una gran contribución en años de esperanza de vida ganados (8).

El procesamiento de la información se realizó con el paquete estadístico SPSS versión 11.5.

\section{Resultados}

La proporción de muertes por causas violentas se incrementó en todas las regiones en ambos sexos entre 1985 y 1999; sin embargo, el incremento fue mayor para los hombres que para las mujeres y la región que presentó la más alta proporción de muertes por estas causas fueron los nuevos departamentos, donde más del $50 \%$ de las muertes corresponden a este grupo. Tanto para los hombres como para las mujeres, las principales causas de muerte en 1985 eran las enfermedades del sistema circulatorio pero disminuyeron en todas las regiones y pasaron a ocupar el segundo lugar en los hombres (cuadro 1).

La tasa de mortalidad masculina se incrementó en casi todas las regiones, excepto en la región oriental; mientras que en las mujeres, disminuyó en todas las regiones. Comparada con la tasa de mortalidad masculina nacional (729 por 100.000 habitantes) (6), en 1999 las regiones atlántica y oriental presentaron una tasa de mortalidad inferior a la nacional (508 y 715 por 100.000 habitantes, respectivamente) mientras que la región central presentó la tasa de mortalidad más alta (951 por 100.000 habitantes). En las mujeres solamente la región atlántica presentó una tasa de mortalidad inferior a la nacional (271 y 352 por 100.000 habitantes, respectivamente); sin embargo, la región en la que más disminuyó fue la oriental (de 457 por 100.000 habitantes pasó a 353) (cuadro 2).

\section{Esperanza de vida temporal}

Debido al incremento en la tasa de mortalidad para los hombres, el cambio en la esperanza de vida temporal fue negativo para las regiones atlántica, central, pacífica y los nuevos departamentos, y positivo, solamente para la región oriental, logrando una ganancia de 0,57 años, que representa el 5,59\% del máximo que se podía lograr; entre las mujeres hubo ganancia en todas 
Cuadro 1. Distribución porcentual de las defunciones según causa, sexo, y región. 15 a 74 años, Colombia $1985,1999$.

\begin{tabular}{|c|c|c|c|c|c|c|c|c|c|c|c|}
\hline \multirow[b]{2}{*}{ Sexo } & \multirow[b]{2}{*}{ Causa } & \multicolumn{5}{|c|}{1985} & \multicolumn{5}{|c|}{1999} \\
\hline & & Atlántica & Oriental & Central & Pacífica & $\begin{array}{l}\text { Nuevos } \\
\text { Deptos. }\end{array}$ & Atlántica & Oriental & Central & Pacífica & $\begin{array}{l}\text { Nuevos } \\
\text { Deptos. }\end{array}$ \\
\hline \multirow{10}{*}{ 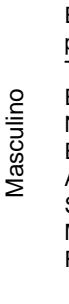 } & $\begin{array}{l}\text { Enfermedades infecciosas y } \\
\text { parasitarias }\end{array}$ & 4,1 & 3,4 & 3,0 & 4,3 & 4,5 & 6.0 & 3,2 & 3,2 & 4.1 & \\
\hline & Tumores (malignos y benignos) & 10,5 & 11,6 & 11,9 & 11,0 & 5,5 & 12,0 & 11,5 & 11,0 & 11,5 & 5,5 \\
\hline & Enfermedades del sistema circulatorio & 30,6 & 26,4 & 23,3 & 24,7 & 12,2 & 23,2 & 22,0 & 17,1 & 18,4 & 11,3 \\
\hline & Neumonía y bronquitis & 2,9 & 4,7 & 4,4 & 4,9 & 2,7 & 3,9 & 5,1 & 5,1 & 4,9 & 2,4 \\
\hline & Enfermedades del sistema digestivo & 3,9 & 4,9 & 3,1 & 4,1 & 2,9 & 3,0 & 4,8 & 2,7 & 3,3 & 1,9 \\
\hline & Accidentes & 14,0 & 17,9 & 17,4 & 13,5 & 16,3 & 11,9 & 13,4 & 9,9 & 10,8 & 12,2 \\
\hline & Suicidios homicidios y otras violencias & 21,6 & 19,6 & 28,4 & 25,0 & 46,4 & 32,7 & 31,1 & 45,0 & 39,0 & 58,7 \\
\hline & Mal definidas & 5,4 & 4,5 & 2,9 & 5,5 & 5,6 & 1,5 & 2,5 & 0,9 & 2,2 & 2,5 \\
\hline & Resto & 7,0 & 7,0 & 5,7 & 7,0 & 3,8 & 5,9 & 6,2 & 5,1 & 5,9 & 2,8 \\
\hline & Total & 100 & 100 & 100 & 100 & 100 & 100 & 100 & 100 & 100 & 100 \\
\hline \multirow{11}{*}{ 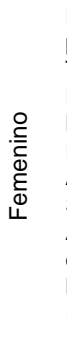 } & $\begin{array}{l}\text { Enfermedades infecciosas y } \\
\text { parasitarias }\end{array}$ & 4,9 & 2,9 & 3,7 & 5,5 & 7,4 & 5,2 & 2,9 & 2,9 & 4,2 & 5,0 \\
\hline & Tumores (malignos y benignos) & 20,5 & 20,5 & 23,3 & 21,0 & 16,0 & 26,1 & 22,6 & 26,5 & 25,3 & 16,1 \\
\hline & Enfermedades del sistema circulatorio & 39,8 & 37,2 & 35,1 & 32,9 & 22,3 & 33,6 & 33,6 & 29,1 & 29,5 & 22,5 \\
\hline & Neumonía y bronquitis & 3,4 & 5,9 & 6,7 & 5,4 & 3,5 & 5,2 & 6,9 & 9,6 & 6,7 & 5,5 \\
\hline & Enfermedades del sistema digestivo & 3,3 & 5,0 & 3,9 & 5,0 & 3,8 & 3,6 & 4,9 & 4,3 & 4,7 & 3,1 \\
\hline & Accidentes & 4,4 & 6,3 & 6,2 & 5,3 & 9,8 & 4,4 & 5,8 & 4,7 & 5,0 & 6,6 \\
\hline & $\begin{array}{l}\text { Suicidios homicidios y otras violencias } \\
\text { Aborto y otras complicaciones del }\end{array}$ & 2,7 & 3,0 & 3,7 & 3,7 & 9,3 & 4,9 & 7,0 & 8,5 & 7,2 & 22,1 \\
\hline & embarazo & 3,2 & 2,1 & 2,0 & 2,4 & 4,6 & 2,6 & 1,4 & 1,3 & 2,0 & 4,0 \\
\hline & Mal definidas & 6,9 & 6,7 & 4,0 & 7,7 & 14,5 & 2,0 & 3,1 & 1,1 & 3,5 & 6,1 \\
\hline & Resto & 10,8 & 10,4 & 11,3 & 11,2 & 8,8 & 12,5 & 11,9 & 12,2 & 11,9 & 9,0 \\
\hline & Total & 100 & 100 & 100 & 100 & 100 & 100 & 100 & 100 & 100 & 100 \\
\hline
\end{tabular}

Fuente: cálculos propios a partir de las defunciones DANE.

Cuadro 2. Tasas de mortalidad por causas, según sexo y región, 15 a 74 años. Colombia 1985, 1999 (Tasas 100.000 habitantes).

\begin{tabular}{|c|c|c|c|c|c|c|c|c|c|c|c|}
\hline \multirow[b]{2}{*}{ Sexo } & \multirow[b]{2}{*}{ Causa } & \multicolumn{5}{|c|}{1985} & \multicolumn{5}{|c|}{1999} \\
\hline & & Atlántica & Oriental & Central & Pacífica & $\begin{array}{l}\text { Nuevos } \\
\text { Deptos. }\end{array}$ & Atlántica & Oriental & Central & Pacífica & $\begin{array}{l}\text { Nuevos } \\
\text { Deptos. }\end{array}$ \\
\hline \multirow{12}{*}{ 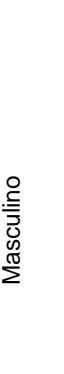 } & $\begin{array}{l}\text { Enfermedades infecciosas y } \\
\text { parasitarias }\end{array}$ & 20 & 26 & 26 & 32 & 35 & 30 & 23 & 31 & 32 & 24 \\
\hline & Tumores (malignos y benignos) & 52 & 89 & 103 & 84 & 43 & 61 & 82 & 104 & 90 & 51 \\
\hline & Enfermedades del sistema & & & & & & & & & & \\
\hline & circulatorio & 151 & 203 & 201 & 187 & 96 & 118 & 157 & 163 & 144 & 105 \\
\hline & Neumonía y bronquitis & 14 & 36 & 38 & 37 & 21 & 20 & 36 & 48 & 38 & 22 \\
\hline & Enfermedades del sistema digestivo & 19 & 38 & 27 & 31 & 23 & 15 & 35 & 25 & 26 & 17 \\
\hline & Accidentes & 69 & 138 & 150 & 103 & 128 & 60 & 96 & 94 & 84 & 114 \\
\hline & Suicidios homicidios y otras & & & & & & & & & & \\
\hline & violencias & 107 & 151 & 245 & 190 & 365 & 166 & 223 & 428 & 305 & 547 \\
\hline & Mal definidas & 27 & 35 & 25 & 42 & 44 & 8 & 18 & 8 & 17 & 24 \\
\hline & Resto & 35 & 54 & 50 & 53 & 30 & 30 & 44 & 49 & 46 & 26 \\
\hline & Total & 494 & 771 & 864 & 759 & 786 & 508 & 715 & 951 & 782 & 932 \\
\hline \multirow{15}{*}{ 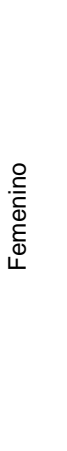 } & $\begin{array}{l}\text { Enfermedades infecciosas y } \\
\text { parasitarias }\end{array}$ & 15 & 13 & 18 & 25 & 25 & 14 & 10 & 12 & 17 & 16 \\
\hline & Tumores (malignos y benignos) & 63 & 94 & 110 & 95 & 53 & 71 & 80 & 111 & 100 & 52 \\
\hline & Enfermedades del sistema & & & & & & & & & & \\
\hline & circulatorio & 122 & 170 & 166 & 148 & 75 & 91 & 119 & 122 & 116 & 72 \\
\hline & Neumonía y bronquitis & 10 & 27 & 32 & 24 & 12 & 14 & 24 & 40 & 26 & 18 \\
\hline & Enfermedades del sistema digestivo & 10 & 23 & 18 & 22 & 13 & 10 & 17 & 18 & 18 & 10 \\
\hline & Accidentes & 13 & 29 & 29 & 24 & 33 & 12 & 20 & 20 & 20 & 21 \\
\hline & Suicidios homicidios y otras & & & & & & & & & & \\
\hline & violencias & 8 & 14 & 17 & 17 & 31 & 13 & 25 & 35 & 29 & 71 \\
\hline & Aborto y otras complicaciones del & & & & & & & & & & \\
\hline & embarazo y parto & 10 & 10 & 9 & 11 & 15 & 7 & 5 & 5 & 8 & 13 \\
\hline & Mal definidas & 21 & 31 & 19 & 35 & 48 & 5 & 11 & 4 & 14 & 20 \\
\hline & Resto & 33 & 47 & 53 & 51 & 29 & 34 & 42 & 51 & 47 & 29 \\
\hline & Total & 305 & 457 & 472 & 451 & 334 & 271 & 353 & 419 & 394 & 321 \\
\hline & Índice de masculinidad & 1,6 & 1,7 & 1,8 & 1,7 & 2,4 & 1,9 & 2,0 & 2,3 & 2,0 & 2,9 \\
\hline
\end{tabular}

Fuente: cálculos propios a partir de las defunciones DANE. 
las regiones, siendo la oriental la que mayor ganancia obtuvo (1,66 años, $27,3 \%$ del máximo que se podía incrementar) (cuadro 3 ).

Los nuevos departamentos presentaron la mayor pérdida en la esperanza de vida temporaria para los hombres (2,04 años, $16,91 \%)$ y la menor ganancia para las mujeres (0,52 años, 8,8\%).

La diferencia entre la esperanza de vida temporaria de los hombres y las mujeres se incrementó en todas las regiones, siendo mayor en la región central (2,5 años) y en los nuevos departamentos (2,56 años).

\section{Contribución de las causas de muerte a la esperanza de vida temporal}

En 1985 las principales causas de muerte para los hombres fueron las enfermedades del sistema circulatorio, las muertes violentas (suicidios, homicidios y otras violencias) y los accidentes. En las regiones atlántica y oriental las enfermedades del sistema circulatorio ocupaban el primer lugar. En 1999 las muertes violentas pasaron a ocupar el primer lugar en todas las regiones. Las regiones que presentaron el mayor incrementó en las tasas de mortalidad por estas causas fueron la región central y los nuevos departamentos ocasionando una pérdida en la esperanza de vida temporal de 2,15 y 2,55 años, respectivamente (cuadro 4).

Las enfermedades del sistema circulatorio, aunque están entre las primeras causas de muerte, disminuyeron en todas las regiones excepto en los nuevos departamentos. La mayor ganancia por la disminución de estas causas la obtuvo la región pacífica ( 0,78 años); en cuanto a las enfermedades infecciosas y parasitarias, la mayor ganancia fue para los nuevos departamentos ( 0,15 años).

Los tumores solamente disminuyeron en la región oriental, logrando una ganancia de 0,07 años; las neumonías y bronquitis aumentaron en todas las regiones, mientras que las enfermedades del sistema digestivo disminuyeron.

Los accidentes disminuyeron en todas las regiones, logrando la mayor ganancia la región central (0,65 años).

Para las mujeres, tanto en 1985 como en 1999 , las principales causas de muerte fueron las enfermedades del sistema circulatorio y los tumores (malignos y benignos) en todas las regiones, excepto en los nuevos departamentos, donde los tumores pasaron al tercer puesto y las muertes violentas al segundo lugar. Se destaca que, aunque las enfermedades del sistema circulatorio son la principal causa de muerte entre las mujeres, éstas disminuyeron en todas las regiones, logrando la mayor ganancia la región central ( 1,20 años).

Las neumonías y bronquitis solamente disminuyeron en la región oriental y las enfermedades del sistema digestivo disminuyeron en todas las regiones.

Las muertes violentas también aumentaron para las mujeres de todas las regiones, pero no en la misma forma que en los hombres, causando una

Cuadro 3. Esperanza de vida temporal, 15 a 74 años. 1985, 1999. Cambio absoluto y relativo por región y sexo.

\begin{tabular}{|c|c|c|c|c|c|c|c|c|}
\hline \multirow{3}{*}{ Región } & \multicolumn{4}{|c|}{ Masculino } & \multicolumn{4}{|c|}{ Femenino } \\
\hline & 1985 & 1999 & $\begin{array}{l}\text { Cambio } \\
\text { absoluto }\end{array}$ & $\begin{array}{l}\text { Cambio } \\
\text { relativo }\end{array}$ & 1985 & 1999 & $\begin{array}{l}\text { Cambio } \\
\text { absoluto }\end{array}$ & $\begin{array}{l}\text { Cambio } \\
\text { relativo }\end{array}$ \\
\hline & $e_{x}$ & $e_{x}$ & ${ }_{n} A C_{x}$ & ${ }_{n} R C_{x}$ & $e_{x}$ & $e_{x}$ & ${ }_{n} A C_{x}$ & ${ }_{n} R C_{x}$ \\
\hline Atlántica & 52,32 & 52,28 & $-0,04$ & $-0,0057$ & 54,92 & 55,96 & 1,04 & 0,2041 \\
\hline Oriental & 49,74 & 50,31 & 0,57 & 0,0559 & 53,92 & 55,58 & 1,66 & 0,2730 \\
\hline Central & 48,05 & 47,01 & $-1,03$ & $-0,0863$ & 53,11 & 54,57 & 1,46 & 0,2116 \\
\hline Pacífica & 49,51 & 49,10 & $-0,41$ & $-0,0388$ & 53,36 & 54,72 & 1,36 & 0,2044 \\
\hline Nuevos departamentos & 47,95 & 45,91 & $-2,04$ & $-0,1691$ & 54,04 & 54,56 & 0,52 & 0,0880 \\
\hline
\end{tabular}

Fuente: cálculos propios a partir de las defunciones DANE. 
Cuadro 4. Cambio en las tasas de mortalidad y su contribución en la esperanza de vida temporal entre 1985 y 1999 , según sexo y región, 15 a 74 años.

\begin{tabular}{|c|c|c|c|c|c|c|c|c|c|c|c|}
\hline \multirow[b]{2}{*}{ Sexo } & \multirow[b]{2}{*}{ Causa } & \multicolumn{5}{|c|}{$\begin{array}{l}\text { Cambio en las tasas de mortalidad } \\
\text { (tasas por } 100.000 \text { habitantes) }\end{array}$} & \multicolumn{5}{|c|}{$\begin{array}{l}\text { Contribución en la esperanza } \\
\text { de vida temporal (años) }\end{array}$} \\
\hline & & Atlántica & Oriental & Central & Pacífica & $\begin{array}{l}\text { Nuevos } \\
\text { deptos. }\end{array}$ & Atlántica & Oriental & Central & Pacífica & $\begin{array}{l}\text { Nuevos } \\
\text { deptos. }\end{array}$ \\
\hline \multirow{10}{*}{ 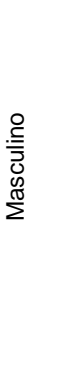 } & $\begin{array}{l}\text { Enfermedades infecciosas y } \\
\text { parasitarias }\end{array}$ & 10 & -3 & 5 & 0 & -11 & $-0,03$ & 0.03 & $-0,06$ & 0.00 & 0,15 \\
\hline & $\begin{array}{l}\text { Tumores (malignos y benignos) } \\
\text { Enfermedades del sistema }\end{array}$ & 9 & -7 & 2 & 6 & 8 & $-0,03$ & 0,07 & $-0,02$ & $-0,11$ & $-0,11$ \\
\hline & circulatorio & -34 & -46 & -38 & -43 & 9 & 0,10 & 0,47 & 0,45 & 0,78 & $-0,13$ \\
\hline & Neumonía y bronquitis & 5 & 0 & 10 & 1 & 1 & $-0,02$ & 0,00 & $-0,12$ & $-0,02$ & $-0,01$ \\
\hline & Enfermedades del sistema digestivo & -4 & -3 & -2 & -6 & -5 & 0,01 & 0,03 & 0,02 & 0,10 & 0,07 \\
\hline & $\begin{array}{l}\text { Accidentes } \\
\text { Suicidios homicidios y otras }\end{array}$ & -9 & -42 & -56 & -19 & -14 & 0,03 & 0,42 & 0,65 & 0,33 & 0,20 \\
\hline & violencias & 59 & 71 & 183 & 115 & 182 & $-0,18$ & $-0,73$ & $-2,15$ & $-2,08$ & $-2,55$ \\
\hline & Mal definidas & -19 & -17 & -16 & -25 & -20 & 0,06 & 0,17 & 0,19 & 0,45 & 0,28 \\
\hline & Resto & -5 & -10 & -1 & -7 & -4 & 0,01 & 0,10 & 0,01 & 0,13 & 0,05 \\
\hline & Total & 15 & -56 & 88 & 23 & 146 & $-0,04$ & 0,57 & $-1,03$ & $-0,41$ & $-2,04$ \\
\hline \multirow{13}{*}{ 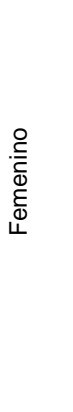 } & Enfermedades infecciosas y & & & & & & $00 ?$ & & & & \\
\hline & $\begin{array}{l}\text { parasitarlas } \\
\text { Tumores (malianos y penianos) }\end{array}$ & -1 & -3 & -5 & -8 & -9 & 0,03 & 0,05 & 0,15 & 0,20 & 0,35 \\
\hline & Enfermedades del sistema & 8 & -14 & 1 & 5 & -2 & $-0,24$ & 0,22 & $-0,03$ & $-0,12$ & 0,07 \\
\hline & circulatorio & -30 & -51 & -44 & -32 & -2 & 0,93 & 0,82 & 1,20 & 0,77 & 0,10 \\
\hline & Neumonía y bronquitis & 4 & -3 & 8 & 2 & 6 & $-0,11$ & 0,04 & $-0,23$ & $-0,05$ & $-0,23$ \\
\hline & Enfermedades del sistema digestivo & 0 & -5 & -1 & -4 & -3 & 0,01 & 0,08 & 0,02 & 0,10 & 0,10 \\
\hline & Accidentes & -2 & -9 & -10 & -4 & -12 & 0,05 & 0,14 & 0,27 & 0,10 & 0,46 \\
\hline & Suicidios homicidios y otras & & & & & & & & & & \\
\hline & $\begin{array}{l}\text { violencias } \\
\text { Aborto y otras complicaciones del }\end{array}$ & 5 & 11 & 18 & 12 & 40 & $-0,15$ & $-0,18$ & $-0,50$ & $-0,28$ & $-1,60$ \\
\hline & embarazo y parto & -2 & -5 & -4 & -3 & -3 & 0,08 & 0,08 & 0,11 & 0,07 & 0,10 \\
\hline & Mal definidas & -16 & -20 & -15 & -21 & -29 & 0,48 & 0,31 & 0,40 & 0,50 & 1,15 \\
\hline & Resto & 1 & -5 & -2 & -4 & 0 & $-0,02$ & 0,09 & 0,06 & 0,09 & 0,01 \\
\hline & Total & -34 & -103 & -53 & -57 & -13 & 1,04 & 1,66 & 1,46 & 1,36 & 0,52 \\
\hline
\end{tabular}

Fuente: cálculos propios a partir de las defunciones DANE.

pérdida en la esperanza de vida temporaria mayor en los nuevos departamentos (1,6 años); los accidentes disminuyeron en todas las regiones pero la mayor ganancia fue para los nuevos departamentos (0,46 años).

En cuanto a la mortalidad materna, disminuyó en todas las regiones logrando el mayor beneficio la región Central ( 0,11 años).

\section{Discusión}

El alto incremento de las tasas de mortalidad masculina y la disminución de las tasas de mortalidad femenina produjeron un incremento en el exceso de mortalidad masculina y un aumento de la brecha entre la esperanza de vida de los hombres y las mujeres en todas las regiones del país.

La principal causa de la pérdida en la esperanza de vida temporal de los 15 a 74 años en ambos sexos, fue el incremento de las defunciones por suicidios, homicidios y otras causas violentas; aunque la pérdida fue mayor para los hombres que para las mujeres. Los más afectados por estas causas fueron los hombres y las mujeres más jóvenes (9-13).

En 1985, la esperanza de vida temporal para los hombres de la región Atlántica fue similar a la de Argentina y la de las mujeres fue similar a la de México; para las otras regiones fue inferior a la de Argentina, Uruguay, Chile y México con diferencias de más de dos años para los hombres y menores de tres años para las mujeres. En 1999, la esperanza de vida temporal fue inferior a la de 1990 de estos países (14).

En general, cuando se diseñan planes para reducir la mortalidad, entre las primeras soluciones que se proponen están los programas de vacunación, ampliar el acceso a los servicios de salud, los programas nutricionales (para madres y niños), y otros que son importantes para lograr mejoras; 
sin embargo, también se debe tener en cuenta que con acciones probablemente más simples se pueden reducir las muertes por violencia (suicidio, homicidio, accidentes) produciendo efectos inmediatos en la esperanza de vida (14).

Se destaca el hecho que los accidentes disminuyeron en todas las regiones entre 1985 y 1999, logrando un aporte positivo en la esperanza de vida temporal, lo que no se hubiera podido mostrar si estas causas se hubieran incluido entre las causas violentas. Aunque la mortalidad por esta causa disminuyó, se deben seguir impulsando las medidas tendientes a la disminución del consumo de alcohol y el uso de cinturones de seguridad por los pasajeros de automóviles y el uso del casco de seguridad en motociclistas, puesto que en los últimos años se ha incrementado el número de motos y automóviles, especialmente en las grandes ciudades del país (15).

Según los resultados de este estudio comparados con los otros estudios realizados en el país, se confirma que el incremento de la mortalidad por causas violentas y la disminución de la mortalidad por otras causas no es el mismo en todas las regiones y que los programas para mejorar la situación de salud y para reducir la mortalidad deben ser acordes con la situación de cada población; se deben generar acciones en salud tendientes a disminuir los factores que producen la violencia, responsable de un alto porcentaje de las muertes causadas por accidentes, violencias y traumatismos (9-13).

Las enfermedades del sistema circulatorio afectan más a las mujeres que a los hombres y, aunque continúan siendo una de las principales causas de mortalidad en Colombia, disminuyeron entre 1985 y 1999 en ambos sexos en casi todas las regiones logrando un aporte positivo en la esperanza de vida temporal.

La ganancia en la esperanza de vida temporal fue mínima puesto que los aportes positivos producidos por la disminución de la mortalidad por causas naturales se anulan con los aportes negativos producidos por el incremento de la mortalidad por suicidios, homicidios y otras violencias que son evitables.

\section{Agradecimientos}

Los autores expresan su agradecimiento a Myriam Ordóñez Gómez, asesora del proyecto, por su valiosa colaboración.

\section{Conflicto de intereses}

Los autores declaran no tener conflicto de intereses con el contenido del manuscrito.

\section{Financiación}

Este estudio fue desarrollado como parte del proyecto 21040412690 financiado por Colciencias y el Instituto Nacional de Salud.

\section{Referencias}

1. Organización Panamericana de la Salud, Conselleria de Sanidade de la Xunta de Galicia. Demografía. En: Epidat v.3. [Consultado: marzo de 2008]. Disponible en: http://dxsp.sergas.es/ApliEdatos/ Epidat/Ayuda/2-Ayuda\%20Demograf\%EDa.pdf

2. Arriaga E. Adult mortality in the era of HIV/AIDS: Latin America and Caribbean. New York: Department of Economic and Social Affairs, Population Division, United Nations Secretariat; 2003.

3. Organización Panamericana de la Salud. La salud en las Américas. Volumen 1. Washington, D. C.: OPS; 2002. p. 13.

4. Balladelli PP. Elementos para establecer prioridades en investigación en salud pública. Bogotá, D.C., 25 de octubre de 2006 [Consultado: junio de 2007] Disponible en: http://www.col.ops-oms.org/docs/Conferencia\%20 COLCIENCIA\%20Prioridades\%20investigaci\%C3 \%B3n\%20SP\%20251006.doc

5. Ordóñez M, Castaño L, Pinilla C, López E. La mortalidad en Colombia en el período 1982-1996. Bogotá, D.C.: Instituto Nacional de Salud, Ministerio de la Protección Social; 2003. [Consultado: febrero 2006]. Disponible en: http://www.ins.gov.co/pdf_investiga/ 2005_ss_mortalidad_general_1982_1996.pdf

6. Arriaga E. Measuring and explaining the change in life expectancies. Demography. 1984;21:83-96

7. Organización Panamericana de la Salud/ Organización Mundial de la Salud. Clasificación Internacional de Enfermedades. Washington, D. C.: OPS; 1975.

8. Organización Panamericana de la Salud/ Organización Mundial de la Salud. Clasificación Estadística internacional de enfermedades y problemas relacionados con la salud, CIE-10. 10ª revisión. Washington, D. C.: OPS; 1995.

9. López E, Arce P. Impacto de las causas de muerte en la esperanza de vida. Colombia, Bogotá, Antioquia y 
Valle, 1985-1999. Boletín Epidemiológico Distrital. 2006;9:2-15.

10. Serna C, Grisales H, Caicedo B, Uribe D. Causas de mortalidad en jóvenes y su contribución al cambio en la esperanza de vida. Bogotá 1989-1999. Boletín Epidemiológico Distrital. 2004;8:2-10.

11. Grisales H, Caicedo B, Serna C, Uribe D. Causas de mortalidad en jóvenes y su contribución al cambio en la esperanza de vida. Cali, 1989-1999. Colomb Med. 2005;36:85-93.

12. Aristizábal E, Posada M, Estrada G, Grisales H. Cambio en la esperanza de vida de la población de Medellín por las seis principales causas de muerte entre 1989-91 y 1994-96. En: Fernández S, Grisales H, editores. Estudios sobre la mortalidad: diferentes enfoques. Medellín: DANE, Universidad de Antioquia; 2003. p. $13-25$.
13. Grisales H, Estrada GS, Aristizábal M, Posada M. Cambio en la esperanza de vida según tres grandes grupos de causas de muerte en Medellín, Colombia, de 1989-1991 a 1994-19996. Rev Panam Salud Pública. 2002;12:305-12.

14. Boleda M, Arriaga EE. América Latina: mortalidad por accidentes y por violencia contra las personas. Notas de población No. 70. CEPAL 2000. p 87-119. [Consultado: marzo de 2006]. Disponible en:_http:// www.eclac.org/publicaciones/xml/6/6776/ lcg21003.pdf.

15. Londoño J, Grisales R, Fernández S, Cadena E, Años de vida saludable perdidos por la población de Medellín. Un análisis especial por homicidio y accidentes de vehículo de motor. Rev Fac Nac Salud Pública. 1999;17:63-92. 